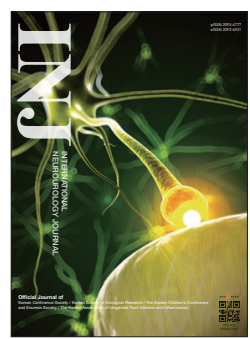

\title{
The Overactive Bladder Symptom Score, International Prostate Symptom Score-Storage Subscore, and Urgency Severity Score in Patients With Overactive Bladder and Hypersensitive Bladder: Which Scoring System is Best?
}

\author{
Fei-Chi Chuang ${ }^{1}$, Sheng-Mou Hsiao ${ }^{2}$, Hann-Chorng Kuo ${ }^{3}$ \\ ${ }^{1}$ Department of Obstetrics and Gynecology, Kaohsiung Chang Gung Memorial Hospital and Chang Gung University College of Medicine, Kaohsiung, Taiwan \\ ${ }^{2}$ Department of Obstetrics and Gynecology, Far Eastern Memorial Hospital, Banqiao, New Taipei, Taiwan \\ ${ }^{3}$ Department of Urology, Buddhist Tzu Chi General Hospital, Buddhist Tzu Chi University, Hualien, Taiwan
}

Purpose: To evaluate the correlations among the Overactive Bladder Symptom Score (OABSS), International Prostate Symptom Score-Storage Subscore (IPSS-S), and the modified Urgency Severity Scale (USS) in patients with overactive bladder $(\mathrm{OAB})$ and hypersensitive bladder (HSB) and to identify the most useful diagnostic tool for classifying the severity of OAB. Methods: We retrospectively reviewed the charts of consecutive patients with OAB who visited our urologic clinics for treatment. All patients underwent a detailed history, physical examination, urinalysis, uroflowmetry, and postvoid residual volume measurement, and completed a 3-day voiding diary. All patients answered the Chinese versions of the IPSS, OABSS, and USS, according to which they were classified as having wet or dry $\mathrm{OAB}$ based on whether their chief complaint was urgency urinary incontinence or urgency without incontinence. HSB was defined as a functional bladder capacity $<350 \mathrm{~mL}$ and a USS of 0 or 1 . Results: The records of $325 \mathrm{OAB}$ patients ( 99 women and $226 \mathrm{men}$ ) were reviewed. The OAB subgroups included HSB $(n=31)$, OAB-dry $(n=74)$, and OAB-wet $(n=220)$. One-way analysis of variance showed significant differences among the $\mathrm{OAB}$ subgroups evaluated using each scoring system. Each scoring system was significantly correlated with the OAB subgroups. The Spearman rho was 0.983 for the USS, 0.651 for the OABSS, and 0.428 for the IPSS-S.

Conclusions: The IPSS-S, OABSS, and USS showed good correlations with the OAB subgroups. Their ranking in terms of discriminant ability for classifying OAB severity as HSB, OAB-dry, and OAB-wet was USS > OABSS > IPSS-S. The simplest survey, the USS, with a single item scored from 0 to 4 , had the strongest correlation with the OAB severity subgroups.

Keywords: Symptom score; Urgency; Overactive bladder; Hypersensitive bladder

- Research Ethics: This study was approved by the Research Ethics Committee of the Buddhist Tzu-Chi General Hospital (TCGH IRB 098-38).

- Conflict of Interest: No potential conflict of interest relevant to this article was reported.

\section{INTRODUCTION}

Overactive bladder $(\mathrm{OAB})$ is defined as a symptom syndrome of urinary urgency, with or without urgency incontinence, usu- ally with urinary frequency, and nocturia, in the absence of infection or other obvious pathological features [1,2]. Patients are classified as having OAB-wet or OAB-dry based on whether their chief complaint is urgency frequency with or without ur-

Corresponding author: Hann-Chorng Kuo (D https://orcid.org/0000-0001-7165-4771 Department of Urology, Buddhist Tzu Chi General Hospital, No. 707, Section 3, Chuang Yang Road, Hualien City, Taiwan

E-mail: hck@tzuchi.com.tw / Tel: +886-3-8561825 (ext. 2117) / Fax: +886-3-8560794

Submitted: February 26, 2016 / Accepted after revision: June 7, 2016 
gency urinary incontinence (UUI), respectively. Urgency is defined as the core symptom of OAB. However, the word "urgen$c y$ " and its definition have been the source of much debate and confusion. Yamaguchi et al. [3] conducted a "patient trust study" in 21 intelligent (i.e., to be "trusted") female patients with OAB. The results showed that in $43 \%$ of patients seeking medical care, urgency episodes occurred less than once per day, and some patients had days without urgency. They hypothesized that OAB may be more accurately defined as hypersensitive bladder (HSB), rather than as a syndrome characterized by urgency. Increased bladder sensitivity or hypersensitivity appears to be the basis for inducing urgency. Lee et al. [4] also reported that bladder sensory profiles indicated that $\mathrm{OAB}$ patients had more sensitive bladders than non-OAB subjects. They concluded that the bladders of OAB patients may be not only overactive, but also hypersensitive. In clinical practice, HSB and OAB symptoms usually overlap, and patients with HSB might be classified as having dry OAB $[5,6]$.

HSB, OAB-dry, and OAB-wet represent the spectrum of $\mathrm{OAB}$ severity. Wet $\mathrm{OAB}$ is usually considered to be the most severe subtype of $\mathrm{OAB}, \mathrm{UUI}$ is stressful in terms of the physical and psychological ability to cope with the requirements of daily life and has a strong negative impact on the quality of a patient's life $[7,8]$. In a large population-based cross-sectional study, Agarwal et al. [9] reported that urinary urgency was the most common troubling symptom. However, for individuals, urgency incontinence was the most likely to be rated as bothersome. UUI is associated with numerous comorbid conditions and inflicts a substantial personal burden on many aspects of patients' lives, such as an increased risk for falls and fractures, depression, and problems with sexual function [8]. Patients with wet $\mathrm{OAB}$ make more adaptation efforts in daily living than patients with dry OAB $[10,11]$.

$\mathrm{OAB}$ is defined by subjective symptoms, rather than objective measures. The patient's perspective is the key point for the management of OAB. Several patient-reported questionnaires have been developed to characterize patients' symptoms and their impact on quality of life $[12,13]$. Some of these questionnaires include the Overactive Bladder Questionnaire, Patient Perception of Bladder Condition [14], the Primary OAB Symptom Questionnaire, Overactive Bladder Symptom Composite Score, Urgency Questionnaire, and the Overactive Bladder Symptom Score (OABSS) [15]. Additionally, bladder diaries can provide a detailed record of information regarding $\mathrm{OAB}$ symptoms [16]. Nonetheless, keeping such a diary is inconve- nient for some patients.

In our daily practice, we have used several different questionnaires to characterize lower urinary tract symptoms. This raises the question of which questionnaire is the best for determining the severity of $\mathrm{OAB}$. The aim of this study was to evaluate the correlations among the International Prostate Symptom ScoreStorage Subscore (IPSS-S) [17,18], the OABSS [19], and the Urgency Severity Scale (USS) [20] in patients with OAB and HSB and to identify the most useful diagnostic tool for classifying the severity of $\mathrm{OAB}$.

\section{MATERIALS AND METHODS}

We retrospectively reviewed the charts of consecutive patients who complained of storage symptoms and visited our urologic clinic for treatment. As routine practice, all patients underwent a detailed clinical evaluation including a history, physical examination, urinalysis, urine culture, uroflowmetry, and postvoid residual volume measurement and completed a 3-day voiding diary recording urgency and UUI episodes. Additionally, all patients responded to the validated Chinese version of IPSS, which included a quality-of-life index, the OABSS, and the USS. An expert nurse carefully instructed the patients on the voiding diary and administered the questionnaires.

The study inclusion criteria were an age of 18 years or more and the ability to complete the serial evaluation measures. Patients with concomitant urinary tract infections, possible neurogenic lesions, urothelial carcinoma, and severe cardiovascular disease were excluded from the study. This study was approved by the Research Ethics Committee of the Buddhist Tzu-Chi General Hospital (TCGH IRB 098-38). Wet OAB was considered to be present if the 3-day voiding diary revealed at least 1 episode of UUI. Dry OAB was suspected when the voiding diary showed no UUI episode but at least 1 urgency episode. HSB was considered to be present when there was no urgency episode in the voiding diary, but the patient's functional bladder capacity was less than $350 \mathrm{~mL}$. An average voiding frequency of fewer than 8 times per day and a functional bladder capacity of more than $350 \mathrm{~mL}$ were considered normal.

The IPSS is composed of 7 questions investigating storage symptoms (questions 2,4 , and 7 ) and voiding symptoms (questions $1,3,5$, and 6). The storage subscore ranges from 0 to 15 , the voiding subscore ranges from 0 to 20 , and the total score ranges from 0 (no symptoms) to 35 (maximal symptoms). A quality-of-life index, with a scale of 0-6 (delighted to terrible), 
Table 1. Urgency Severity Scale questionnaire

How do you feel before each void? Please record the score that best describes how you feel before voiding. Record at each voiding in the diary according to the following descriptions.

Score
$\begin{aligned} & \text { No feeling of urgency: I can continue activities until I choose to use the bathroom. } \\ & 1\end{aligned}$ Mild feeling of urgency: I can feel the need to urinate, but it is easily tolerated. I can finish my activity or task before going to the bathroom.
$2 \quad \begin{aligned} & \text { Moderate feeling of urgency: The sensation of urgency causes a compelling desire to void. I need to stop my activity or task and go to the } \\ & \text { bathroom. }\end{aligned}$
Severe feeling of urgency: The sensation of urgency causes a compelling desire to void. I have difficulty holding urine. I have to my stop
activity or task to avoid a wetting accident and hurry to the bathroom.

is also included in the IPSS [18].

The OABSS is a symptom assessment questionnaire designed to quantify OAB symptoms into a single score [19,21]. The questionnaire consists of 4 questions on $\mathrm{OAB}$ symptoms with maximum scores ranging from 2 to 5 : daytime frequency (2 points), night-time frequency (3 points), urgency ( 5 points), and UUI ( 5 points). The total score ranges from 0 to 15 points, with higher scores indicating higher symptom severity.

The validated Chinese version of the USS questionnaire is a modified version of the Indevus Urgency Severity Scale (IUSS) [20]. The USS differs from the IUSS because urine leakage is included in the 3 other urgency scales. The USS is scored as 0 (no feeling of urgency), 1 (mild urgency), 2 (moderate urgency), 3 (severe urgency), or 4 (inability to hold urine). We used the validated Chinese version of the USS to distinguish true urgency (USS $=2,3$, or 4 ) from strong urge to void (USS $=0$ or 1 ) for patient selection (Table 1). The USS was administered to all patients by a well-trained nurse who explained the meaning of urgency. The USS questionnaire was validated in terms of test-retest reliability and correlated with a Chinese version of the OABSS to assess its feasibility for patient acceptance and detecting clinical responsiveness to antimuscarinic therapy [22]. The sensation of urgency was defined as the sensation of a strong desire to void that was difficult to defer. When a patient had even a single drop of urine leakage in association with a strong urgency sensation, UUI was considered to have occurred.

The statistical analysis was performed using IBM SPSS Statistics ver. 20.0 (IBM Corp., Armonk, NY, USA). Continuous variables are presented as means \pm standard deviations and categorical data are given as numbers (n) and percentages (\%). Statistical comparisons between the groups were made using 1-way analysis of variance (ANOVA) with multiple comparisons and the
Spearman correlation coefficient rho $(\rho)$ with a subsequent significance test. We described the strength of the correlation using the following guide for the absolute value of rho $(\rho)$ : very weak (0.00-0.19), weak (0.20-0.39), moderate (0.40-0.59), strong (0.60-0.79), and very strong (0.80-1.00). P-values less than 0.05 were considered to indicate statistical significance.

Receiver operating characteristic (ROC) curve analysis was also performed to determine the value of the questionnaire as a diagnostic tool. Using Hosmer and Lemeshow's rule for logistic models, the discriminative abilities of the models were classified according to the area under the ROC curves (AUC or AUROC) as poor $(0.5 \leq \mathrm{AUC}<0.7)$, acceptable $(0.7 \leq \mathrm{AUC}<0.8)$, excellent $(0.8 \leq \mathrm{AUC}<0.9)$, or outstanding $(\mathrm{AUC} \geq 0.9)$ [23]. Comparisons of the AUROC values for each scoring system in each subgroup of $\mathrm{OAB}$ were also made.

\section{RESULTS}

A total of $325 \mathrm{OAB}$ patients were recruited, including 99 women and 226 men. Their mean age was $72.6 \pm 12.0$ years (female, $68.5 \pm 13.7$ years and male, $74.4 \pm 10.8$ years). Thirty-one patients (9.5\%) had HSB, 74 (22.8\%) had OAB-dry, and 220 (67.7\%) had OAB-wet. Table 2 and Fig. 1 show the USS, OABSS, and IPSS-S scores for each of the OAB subgroups (HSB, OAB-dry, and OAB-wet). The scores of each of the questionnaires progressively increased as severity increased from HSB to OAB-dry and OAB-wet. One-way ANOVA showed significant differences among the $\mathrm{OAB}$ severity subgroups evaluated with each scoring system (all $\mathrm{P}<0.001$ ). Multiple comparisons also indicated significant differences among each of the 3 OAB severity subgroups evaluated with each scoring system (all $\mathrm{P}<0.05$ ). Post hoc tests for the USS yielded results of 
Table 2. Comparisons of USS, OABSS, and IPSS-S scores among the OAB subgroups

\begin{tabular}{lccccccc}
\hline OAB subgroup & No. $(\%)$ & $\begin{array}{c}\text { USS } \\
(\text { score 0-4) }\end{array}$ & P-value & $\begin{array}{c}\text { OABSS } \\
(\text { score 0-15) }\end{array}$ & P-value & $\begin{array}{c}\text { IPSS-S } \\
(\text { score 0-15) }\end{array}$ & $\begin{array}{c}\text { P-value } \\
\text { HSB }\end{array}$ \\
\hline $31(9.5)$ & $0.32 \pm 0.48$ & $<0.001^{\text {a) }}$ & $3.16 \pm 1.00$ & $0.002^{\text {a) }}$ & $4.61 \pm 2.19$ & $0.030^{\text {a) }}$ \\
OAB-dry & $74(22.8)$ & $2.57 \pm 0.53$ & $<0.001^{\text {b) }}$ & $5.31 \pm 2.26$ & $<0.001^{\text {b) }}$ & $6.32 \pm 2.74$ & $<0.001^{\text {b) }}$ \\
OAB-wet & $220(67.7)$ & $4.00 \pm 0.07$ & $<0.001^{\text {c) }}$ & $9.44 \pm 3.10$ & $<0.001^{\text {c) }}$ & $8.75 \pm 3.19$ & $<0.001^{\text {c) }}$ \\
\hline
\end{tabular}

Values are presented as mean \pm standard deviation unless otherwise indicated. The P-value is determined with 1-way analysis of variance by linear contrast.

USS, Urgency Severity Scale; OABSS, Overactive Bladder Symptom Score; IPSS-S, International Prostate Symptom Score-Storage Subscore; OAB, overactive bladder; HSB, hypersensitive bladder.

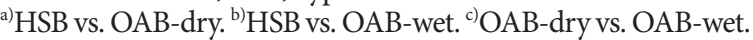
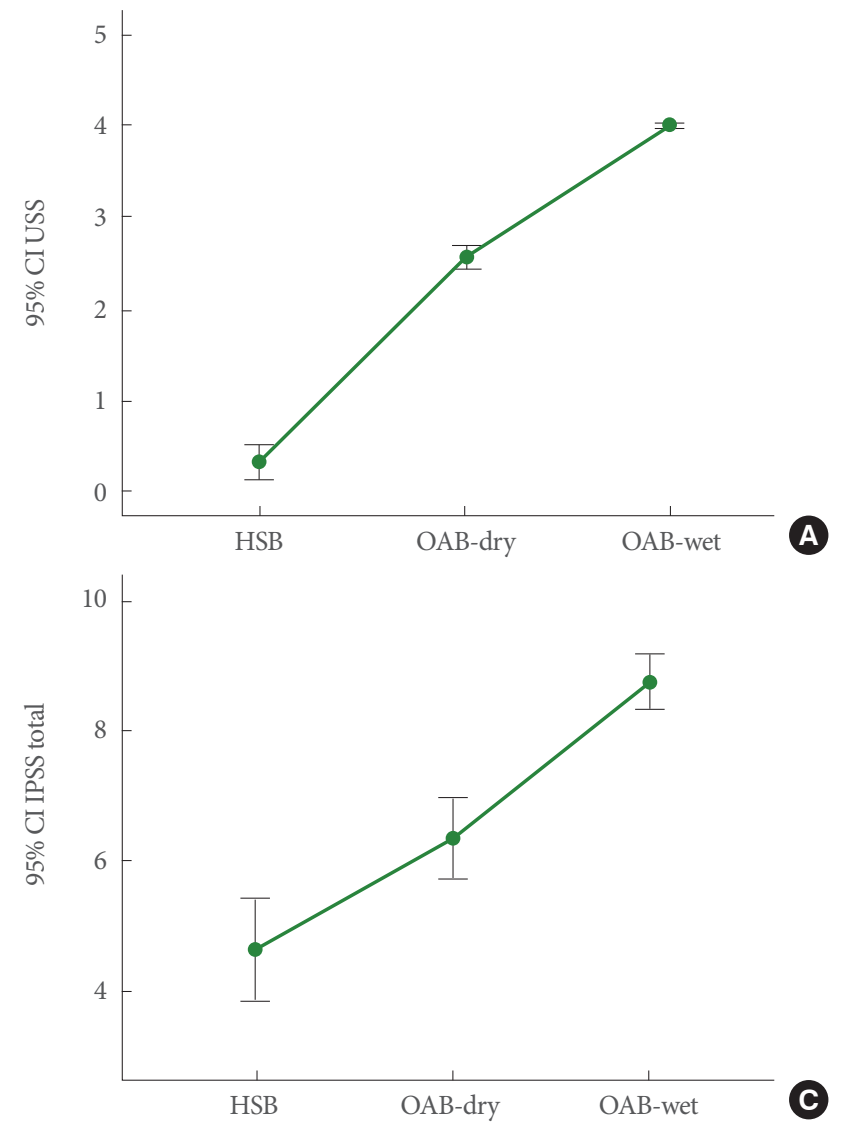

Table 3. Correlation of the OAB subgroups with USS, OABSS, and IPSS-S

\begin{tabular}{lccc}
\hline OAB subgroup & $\begin{array}{c}\text { USS } \\
(\text { score } 0-4)\end{array}$ & $\begin{array}{c}\text { OABSS } \\
(\text { score 0-15) }\end{array}$ & $\begin{array}{c}\text { IPSS-S } \\
(\text { score 0-15) }\end{array}$ \\
\hline Rho $(\rho)$ & 0.983 & 0.651 & 0.428 \\
P-value & $<0.001$ & $<0.001$ & $<0.001$ \\
\hline
\end{tabular}

Rho $(\rho)$ is the Spearman correlation coefficient. The P-value was determined by a subsequent significance test.

$\mathrm{OAB}$, overactive bladder; HSB, hypersensitive bladder; USS, Urgency Severity Scale; OABSS, Overactive Bladder Symptom Score; IPSS-S, International Prostate Symptom Score-Storage Subscore.

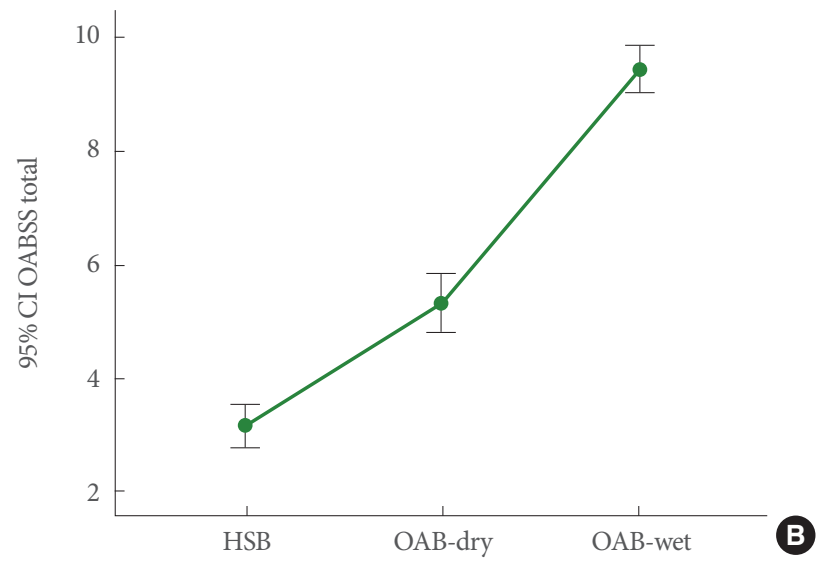

Fig. 1. Distribution of OAB subgroup scores according to the USS (A), OABSS (B), and IPSS-S (C) questionnaires. OAB, overactive bladder; USS, Urgency Severity Scale; OABSS, Overactive Bladder Symptom Score; IPSS-S, International Prostate Symptom Score-Storage Subscore; CI, confidence interval; HSB, hypersensitive bladder.

$\mathrm{P}<0.001$ for all comparisons of OAB subgroups; for the OABSS, $\mathrm{P}=0.002$ for $\mathrm{HSB}$ versus $\mathrm{OAB}$-dry and $\mathrm{P}<0.001$ for the other comparisons; and for the IPSS-S, $\mathrm{P}=0.030$ for HSB versus $\mathrm{OAB}$-dry and $\mathrm{P}<0.001$ for the other comparisons.

Table 3 shows that the scoring system of each questionnaire had a significant correlation with each OAB subgroup (all $\mathrm{P}<0.001$ ). The Spearman correlation coefficients (rho) for the USS, OABSS, and IPSS-S were 0.983 (very strong), 0.651 (strong), and 0.428 (moderate), respectively. Fig. 2 shows the areas under the ROC curve for each scoring system and each 

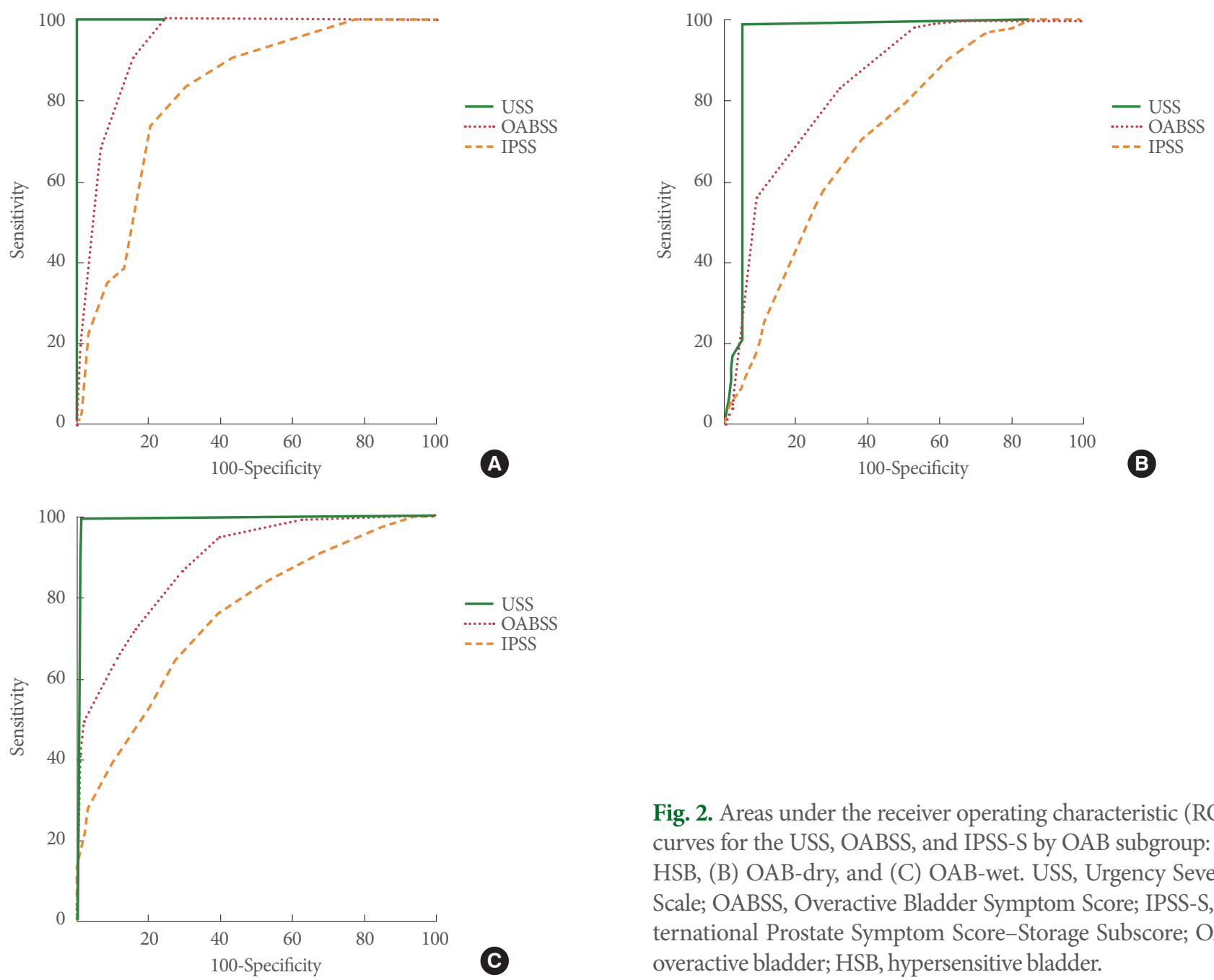

Fig. 2. Areas under the receiver operating characteristic (ROC) curves for the USS, OABSS, and IPSS-S by OAB subgroup: (A) HSB, (B) OAB-dry, and (C) OAB-wet. USS, Urgency Severity Scale; OABSS, Overactive Bladder Symptom Score; IPSS-S, International Prostate Symptom Score-Storage Subscore; OAB, overactive bladder; HSB, hypersensitive bladder.

Table 4. The values and comparisons of the AUC for each questionnaire scoring system by the OAB severity subgroup

\begin{tabular}{|c|c|c|c|c|c|c|}
\hline \multirow{2}{*}{ Variable } & \multicolumn{2}{|l|}{ HSB } & \multicolumn{2}{|c|}{ OAB-dry } & \multicolumn{2}{|c|}{ OAB-wet } \\
\hline & AUC (95\% CI) & $\mathrm{P}$-value & AUC (95\% CI) & $\mathrm{P}$-value & AUC (95\% CI) & P-value \\
\hline USS & $1.000(0.989-1.000)$ & $<0.001^{\mathrm{a})}$ & $0.953(0.924-0.973)$ & $<0.001^{\mathrm{a})}$ & $0.994(0.979-0.999)$ & $<0.001^{\mathrm{a})}$ \\
\hline OABSS & $0.935(0.902-0.959)$ & $<0.001^{\mathrm{b})}$ & $0.842(0.797-0.880)$ & $<0.001^{\text {b) }}$ & $0.888(0.849-0.920)$ & $<0.001^{\text {b) }}$ \\
\hline IPSS-S & $0.815(0.769-0.856)$ & $<0.001^{\mathrm{c})}$ & $0.712(0.659-0.760)$ & $<0.001^{\mathrm{c})}$ & $0.751(0.700-0.797)$ & $<0.001^{c)}$ \\
\hline
\end{tabular}

The P-value was determined with the comparisons of the areas under ROC curve for each scoring systems in each OAB subgroup. AUC, area under receiver operating characteristic curve; OAB, overactive bladder; HSB, hypersensitive bladder; CI, confidence interval; USS, Urgency Severity Scale; OABSS, Overactive Bladder Symptom Score; IPSS-S, International Prostate Symptom Score-Storage Subscore. ${ }^{\text {a) } U S S ~ v s . ~ O A B S S . ~}{ }^{\text {b) }}$ USS vs. IPSS-S. ${ }^{\text {c) } O A B S S}$ vs. IPSS-S.

OAB subgroup. Fig. $2 \mathrm{~A}-\mathrm{C}$ illustrates that the ranking of the AUROC for each OAB subgroup was USS $>$ OABSS $>$ IPSS-S. Table 4 provides the values and comparisons of the AUROC values for each scoring system as related to each $\mathrm{OAB}$ subgroup. The AUROC values indicated that the USS was an outstanding discrim- inative tool for each OAB subgroup (AUROC values of 1.000, 0.953, and 0.994 for HSB, OAB-dry, and OAB-wet, respectively). The OABSS was an outstanding discriminative tool for HSB (0.935), OAB-dry (0.842), and OAB-wet (0.888). The IPSS-S was an excellent discriminative tool for HSB (0.815) and was ac- 
ceptable for OAB-dry (0.712) and OAB-wet (0.751). All comparisons of the AUROC values of each scoring system for each $\mathrm{OAB}$ subgroup showed significant differences (all $\mathrm{P}<0.001$ ).

The USS, OABSS, and IPSS-S correlated well. The correlation coefficients were 0.628 between the USS and the OABSS-total $(\mathrm{P}<0.001), 0.682$ between the OABSS-total and the IPSS-S total $(\mathrm{P}<0.001)$, and 0.340 between the USS and IPSS-S total $(\mathrm{P}<0.001)$. Table 5 presents the correlation coefficients between the $\mathrm{OAB}$ scoring systems and single items. For the OABSS, OABSS-urgency and OABSS-UUI had a stronger correlation with OABSS

Table 5. Correlation among $3 \mathrm{OAB}$ scoring systems

\begin{tabular}{|c|c|c|c|c|c|c|}
\hline \multirow{2}{*}{ Variable } & \multicolumn{2}{|c|}{ USS total } & \multicolumn{2}{|c|}{ OABSS total } & \multicolumn{2}{|c|}{ IPSS-S total } \\
\hline & Rho $(\rho)$ & $\mathrm{P}$-value & Rho $(\rho)$ & P-value & Rho $(\rho)$ & P-value \\
\hline \multicolumn{7}{|l|}{ OABSS } \\
\hline Total & 0.628 & $<0.001$ & - & - & 0.682 & $<0.001$ \\
\hline Freque & 0.039 & 0.483 & 0.300 & $<0.001$ & 0.509 & $<0.001$ \\
\hline Nocts & 0.080 & 0.152 & 0.276 & $<0.001$ & 0.400 & $<0.001$ \\
\hline Urgency & 0.587 & $<0.001$ & 0.907 & $<0.001$ & 0.491 & $<0.001$ \\
\hline UUI & 45 & $<0.0$ & 0.892 & $<0.001$ & 0.536 & $<0.001$ \\
\hline \multicolumn{7}{|l|}{ IPSS-S } \\
\hline Total & 0.340 & $<0.001$ & 0.682 & $<0$. & - & - \\
\hline Freque & 0.086 & 0.123 & 0.317 & $<0.001$ & 0.629 & $<0.001$ \\
\hline Noctu & 0.065 & 0.244 & 0.263 & $<0.001$ & 0.474 & $<0.001$ \\
\hline Urgency & 0.581 & $<0.001$ & 0.748 & $<0.001$ & 0.605 & $<0.001$ \\
\hline
\end{tabular}

Rho $(\rho)$ is the Spearman correlation coefficient. The P-value was determined by a subsequent significance test.

OAB, overactive bladder; USS, Urgency Severity Scale; OABSS, Overactive Bladder Symptom Score; IPSS-S, International Prostate Symptom Score-Storage Subscore; UUI, urgency urinary incontinence. -total than OABSS-frequency and OABSS-nocturia did. For the single items, only OABSS-urgency, OABSS-UUI, and IPSS-urgency had a significant correlation with the USS $(\mathrm{P}<0.001)$.

Table 6 shows the relationships of USS scores with OABSS and IPSS-S scores. Interestingly, among patients with a USS of 0 , 1 , or 2, the OABSS-total, IPSS-S total, and the single items on the OABSS and IPSS-S did not differ significantly. However, among patients with a USS of 3 or 4 , the OABSS-total and IPSStotal were significantly higher than among patients with a USS of less than 3. Moreover, OABSS-frequency, OABSS-nocturia, IPSS-frequency, and IPSS-nocturia did not differ significantly between patients with a USS of 3 or more and those with a USS of less than 3.

\section{DISCUSSION}

The OABSS, IPSS-S, and USS questionnaires correlated well with the $\mathrm{OAB}$ subgroups. The ranking of these questionnaires in terms of discriminant ability for characterizing the severity of $\mathrm{OAB}$ as HSB, OAB-dry, and OAB-wet was USS > OABSS > IPSS$S$. One possible reason that the USS outperformed the other questionnaires could have been because the USS has only 1 item for patients to score, whereas the OABSS is the sum of 4 items (frequency, nocturia, urgency, and UUI). The IPSS-S is the sum of 3 items: frequency, nocturia, and urgency. Patients could introduce bias into the assessments of their OAB symptoms.

For wet $\mathrm{OAB}$ and dry $\mathrm{OAB}$, patients easily distinguish between episodes of UUI (USS $=4$ ), intolerable urgency (USS $=3$ ),

Table 6. Relationships of USS scores to OABSS and IPSS-S scores

\begin{tabular}{lccccc}
\hline Variable & \multicolumn{3}{c}{ USS } \\
\cline { 2 - 5 } & $0(\mathrm{n}=21)$ & $1(\mathrm{n}=10)$ & $2(\mathrm{n}=33)$ & $3(\mathrm{n}=41)$ & \\
\hline OABSS & & & & \\
Total & $3.24 \pm 0.83$ & $2.90 \pm 1.20$ & $3.97 \pm 1.65$ & $6.29 \pm 2.05^{*}$ & $9.40 \pm 3.07^{*}$ \\
Frequency & $0.62 \pm 0.67$ & $0.30 \pm 0.68$ & $0.94 \pm 0.83$ & $0.90 \pm 0.74$ & $0.76 \pm 0.76$ \\
Nocturia & $2.57 \pm 0.75$ & $2.40 \pm 0.70$ & $2.61 \pm 0.66$ & $2.63 \pm 0.54$ & $2.68 \pm 0.58$ \\
Urgency & 0 & $0.30 \pm 0.48$ & $0.39 \pm 1.20$ & $2.27 \pm 1.58^{*}$ & $2.98 \pm 1.47^{*}$ \\
UUI & $0.05 \pm 0.05$ & 0 & $0.03 \pm 0.17$ & $0.59 \pm 0.89^{*}$ & $3.02 \pm 1.43^{*}$ \\
IPSS & & & & & \\
Total & $5.9 \pm 4.7$ & $4.2 \pm 2.66$ & $5.88 \pm 2.36$ & $6.88 \pm 2.59^{*}$ & \\
Frequency & $1.14 \pm 1.91$ & $0.9 \pm 1.60$ & $2.55 \pm 2.17$ & $2.56 \pm 2.03$ & $2.03 \pm 3.24^{*}$ \\
Nocturia & $3.43 \pm 1.54$ & $2.9 \pm 1.37$ & $3.21 \pm 1.39$ & $3.17 \pm 1.14$ & $3.47 \pm 1.26$ \\
Urgency & $0.05 \pm 0.22$ & $0.10 \pm 0.32$ & $0.06 \pm 0.24$ & $1.17 \pm 1.63^{*}$ & $3.00 \pm 1.66^{*}$ \\
\hline
\end{tabular}

Values are presented as mean \pm standard deviation.

USS, Urgency Severity Scale; OABSS, Overactive Bladder Symptom Score; IPSS-S, International Prostate Symptom Score-Storage Subscore; UUI, urgency urinary incontinence.

*Significantly higher than USS $<3($ all $\mathrm{P}<0.05)$. 
and tolerable urgency (USS =2). Patients with HSB may report mild urgency (USS $=1$ ) or frequency without urgency $(\mathrm{USS}=0$ ). Because frequency and nocturia are often closely associated with urgency, the scores for frequency and nocturia do not contribute to the severity of OAB. As shown in Table 5, OABSS-total showed the closest correlations with OABSS-urgency and OABSS-UUI, but it was only mildly associated with OABSSfrequency and nocturia. We previously analyzed the correlation between the USS and the OABSS [19]. The main contributions to the OABSS in patients with a low USS were the day- and night-time urination frequencies. The contribution of urgency and UUI became significant in patients with high urgency scores [24]. The findings of the present study concurred with those previous findings. The data imply that frequency and nocturia are common symptoms in all OAB subgroups with varying severity. Only patients with intolerable urgency (USS =3) and urgency with leaking (USS $=4$ ) had distinctive OAB symptoms. Therefore, urgency severity (mild, moderate, severe, leakage) alone may be used as a grading system for assessing $\mathrm{OAB}$ severity without including scores for frequency and nocturia.

The OABSS is widely used to identify patients with OAB and to evaluate the severity of $\mathrm{OAB}$, as well as treatment outcomes $[19,21]$. This scoring system is rational and involves all OAB symptoms. The OABSS places different weights on the severity of OAB symptoms of frequency (2 points), nocturia (3 points), urgency (5 points), and UUI (5 points). However, when we correlated the OABSS-total with single items, we found that the correlation was highly significant only for OABSS-urgency $(\rho=0.907)$ and OABSS-UUI $(\rho=0.892)$. OABSS-frequency $(\rho=0.300)$ and OABSS-nocturia $(\rho=0.276)$ were weakly associated with OABSS-total (Table 5). These data indicate that the single item of OABSS-urgency adequately reflected the severity of OAB. Furthermore, because urgency and UUI have a score of 5 points each, the weight of these scores contributes more to the OABSS-total score than items with lesser values.

The IPSS-S system does not have a rating for UUI, so it is difficult to distinguish between wet $\mathrm{OAB}$ and dry $\mathrm{OAB}$ with intolerable urgency. In addition, the scores for the 3 items (frequency, nocturia, urgency) have equal weighting. Therefore, IPSSfrequency and IPSS-nocturia make a meaningful contribution to the IPSS-total score. As shown in Table 5, the correlation coefficients between the IPSS-S total and its 3 items were similar ( $\rho=0.629, \rho=0.474$, and $\rho=0.605$, respectively; all $\mathrm{P}<0.001$ ). However, only the IPSS-urgency and IPSS-S total scores were significantly correlated with the USS ( $\rho=0.581$ and $\rho=0.340$, respectively; both $\mathrm{P}<0.001)$. Moreover, the correlation was more significant for the IPSS-urgency score than for the IPSS-S total score. A similar relationship was found for the correlation between the IPSS-S and OABSS systems. These data confirm the suitability of the definition of $\mathrm{OAB}$ recommended by the International Continence Society. OAB should be defined as a symptom syndrome of urinary urgency, with or without urgency incontinence, usually with urinary frequency and nocturia [25]. Urgency is the core symptom of OAB $[2,10]$. Therefore, the severity of $\mathrm{OAB}$ can be rationally classified according to urgency only. For OAB patients, the most bothersome symptom is urgency or UUI when present. The primary limitation of this study is that the relatively high proportion of wet $\mathrm{OAB}$ patients (67.7\%) may have been a confounding factor.

In conclusion, the simplest questionnaire for the characterization of OAB is the USS (with a scale ranging from 0 to 4 ), which was found to have the closest correlation with the $\mathrm{OAB}$ severity subgroups. The USS alone appears to be adequate for assessing the severity of $\mathrm{OAB}$.

\section{ACKNOWLEDGMENTS}

Ms. Huei-Ling Tseng for organizing materials to the manuscript. Ms. Chih-Yun Lin for assisting statistical analysis.

\section{AUTHOR CONTRIBUTION STATEMENT}

- Full access to all the data in the study and takes responsibility for the integrity of the data and the accuracy of the data analysis: HC Kuo

- Study concept and design: HC Kuo

- Acquisition of data: HC Kuo

-Analysis and interpretation of data: HC Kuo, FC Chuang, SM Hsiao

- Drafting of the manuscript: FC Chuang

- Critical revision of the manuscript for important intellectual content: HC Kuo

- Statistical analysis: FC Chuang, SM Hsiao

- Obtained funding: HC Kuo

- Administrative, technical, or material support: FC Chuang, SM Hsiao

- Study supervision: HC Kuo 


\section{REFERENCES}

1. Abrams P, Cardozo L, Fall M, Griffiths D, Rosier P, Ulmsten U, et al. The standardisation of terminology in lower urinary tract function: report from the standardisation sub-committee of the International Continence Society. Urology 2003;61:37-49.

2. Abrams P, Chapple CR, Jünemann KP, Sharpe S. Urinary urgency: a review of its assessment as the key symptom of the overactive bladder syndrome. World J Urol 2012;30:385-92.

3. Yamaguchi O, Honda K, Nomiya M, Shishido K, Kakizaki H, Tanaka $\mathrm{H}$, et al. Defining overactive bladder as hypersensitivity. Neurourol Urodyn 2007;26(6 Suppl):904-7.

4. Lee SR, Kim HJ, Kim A, Kim JH. Overactive bladder is not only overactive but also hypersensitive. Urology 2010;75:1053-9.

5. Chuang FC, Liu HT, Wang LY, Kuo HC. Overactive bladder changes with time: a 5-year longitudinal followup of changes in overactive bladder symptoms, urodynamic studies and urinary nerve growth factor levels. J Urol 2014;192:458-63.

6. Homma Y. Hypersensitive bladder: a solution to confused terminology and ignorance concerning interstitial cystitis. Int J Urol 2014;21 Suppl 1:43-7.

7. Palmtag H. The patient's perspective: redefining end points. Urology 2004;64(6 Suppl 1):17-20.

8. Coyne KS, Wein A, Nicholson S, Kvasz M, Chen CI, Milsom I. Comorbidities and personal burden of urgency urinary incontinence: a systematic review. Int J Clin Pract 2013;67:1015-33.

9. Agarwal A, Eryuzlu LN, Cartwright R, Thorlund K, Tammela TL, Guyatt $\mathrm{GH}$, et al. What is the most bothersome lower urinary tract symptom? Individual- and population-level perspectives for both men and women. Eur Urol 2014;65:1211-7.

10. Hung MJ, Ho ES, Shen PS, Sun MJ, Lin AT, Chen GD, et al. Urgency is the core symptom of female overactive bladder syndrome, as demonstrated by a statistical analysis. J Urol 2006;176:636-40.

11. Stewart WF, Van Rooyen JB, Cundiff GW, Abrams P, Herzog AR, Corey $\mathrm{R}$, et al. Prevalence and burden of overactive bladder in the United States. World J Urol 2003;20:327-36.

12. Matza LS, Thompson CL, Krasnow J, Brewster-Jordan J, Zyczynski T, Coyne KS. Test-retest reliability of four questionnaires for patients with overactive bladder: the overactive bladder questionnaire (OAB-q), patient perception of bladder condition (PPBC), urgency questionnaire (UQ), and the primary $\mathrm{OAB}$ symptom questionnaire (POSQ). Neurourol Urodyn 2005;24:215-25.

13. Starkman JS, Dmochowski RR. Urgency assessment in the evaluation of overactive bladder (OAB). Neurourol Urodyn 2008;27:1321.
14. Coyne KS, Matza LS, Kopp Z, Abrams P. The validation of the patient perception of bladder condition (PPBC): a single-item global measure for patients with overactive bladder. Eur Urol 2006;49:107986.

15. Homma Y, Yoshida M, Seki N, Yokoyama O, Kakizaki H, Gotoh M, et al. Symptom assessment tool for overactive bladder syndrome-overactive bladder symptom score. Urology 2006;68:318-23.

16. Brown JS, McNaughton KS, Wyman JF, Burgio KL, Harkaway R, Bergner D, et al. Measurement characteristics of a voiding diary for use by men and women with overactive bladder. Urology 2003;61: 802-9.

17. Hsiao SM, Lin HH, Kuo HC. International Prostate Symptom Score for assessing lower urinary tract dysfunction in women. Int Urogynecol J 2013;24:263-7.

18. Scarpero HM, Fiske J, Xue X, Nitti VW. American Urological Association Symptom Index for lower urinary tract symptoms in women: correlation with degree of bother and impact on quality of life. Urology 2003;61:1118-22.

19. Hung MJ, Chou CL, Yen TW, Chuang YC, Meng E, Huang ST, et al. Development and validation of the Chinese Overactive Bladder Symptom Score for assessing overactive bladder syndrome in a RESORT study. J Formos Med Assoc 2013;112:276-82.

20. Chung SD, Liao CH, Chen YC, Kuo HC. Urgency severity scale could predict urodynamic detrusor overactivity in patients with overactive bladder syndrome. Neurourol Urodyn 2011;30:1300-4.

21. Homma Y, Kakizaki H, Yamaguchi O, Yamanishi T, Nishizawa O, Yokoyama $\mathrm{O}$, et al. Assessment of overactive bladder symptoms: comparison of 3-day bladder diary and the overactive bladder symptoms score. Urology 2011;77:60-4.

22. Chou EC, Hung MJ, Yen TW, Chuang YC, Meng E, Huang ST, et al. The translation and validation of Chinese overactive bladder symptom score for assessing overactive bladder syndrome and response to solifenacin treatment. J Formos Med Assoc 2014;113:506-12.

23. Hosmer DW, Lemeshow S. Assessing the fit of the model. In: Hosmer DW, Lemeshow S. Applied logistic regression. New York: John Wiley and Sons; 2000. p. 143.

24. Ke QS, Kuo HC. Strong correlation between the overactive bladder symptom score and urgency severity score in assessment of patients with overactive bladder syndrome. Tzu Chi Med J 2010;22: 82-6.

25. Abrams P, Cardozo L, Fall M, Griffiths D, Rosier P, Ulmsten U, et al. The standardisation of terminology of lower urinary tract function: report from the Standardisation Sub-committee of the International Continence Society. Am J Obstet Gynecol 2002;187:11626. 